\title{
Congestion management in the deregulated electricity market: an assessment of locational pricing, redispatch and regulation
}

\author{
R. M. Hermans ${ }^{\# 1}$, P. P. J. Van den Bosch ${ }^{\# 2}$, A. Jokić ${ }^{\# 3}$, P. Giesbertz ${ }^{* 4}$, P. Boonekamp ${ }^{\ddagger 5}$, A. Virag $\# 6$ \\ \# Dept. Electrical Engineering, Eindhoven University of Technology, P.O. Box 513, 5600 MB Eindhoven, NL \\ 1,2,3,6 \{r.m.hermans, p.p.j.v.d.bosch, a.jokic, a.virag\}etue.nl \\ * Statkraft Markets B.V., Gustav Mahlerplein 100, 1082 MA Amsterdam, NL \\ ${ }^{4}$ Paul. Giesbertzastatkraft.com \\ $\ddagger$ Amsterdam Power Exchange (APX), Strawinskylaan 729, 1077 XX Amsterdam, NL \\ ${ }^{5} \mathrm{P}$. Boonekamp@apxendex. com
}

\begin{abstract}
We analyze the fundamental differences between locational pricing and redispatch-based congestion management, followed by an assessment of their effects on grid operation and market efficiency. It is indicated that although optimal nodal pricing and congestion redispatch can provide equal results in terms of power injections, they are not equivalent in terms of short-run social welfare. Moreover, a modeling framework is presented to decouple and analyze the effects of transmission system operator/regulator and prosumer behavior on energy market efficiency in a transparent fashion. All results are illustrated on the basis of case studies for the IEEE 39-bus New England test network.
\end{abstract}

\section{INTRODUCTION}

Finite transmission-line capacity and a lack of powerflow controllability pose strict constraints on electrical power transmission. Persistent violation of network constraints can cause power outages with severe economical consequences. Accordingly, congestion management is vital to guarantee secure operation of the electricity grid.

Before liberalization of the electricity market, network limitations could effectively be taken into account during the dispatch phase, which was centrally coordinated and involved a small number of cooperating parties only. At present, power networks are deregulated and decentralized, and their operation relies on providing market participants with proper incentives for societal beneficial behavior. When congestion management is the main focus, transmission system operators (TSOs) need to design arrangements that maximize market performance while simultaneously motivating producers and consumers to adapt to network restrictions. It is well-known that transmission constraints can induce market inefficiency, because congestion can supply participants with large market power. To prevent this, market-based congestion management should be robust, fair and transparent.

In Europe, two different congestion management schemes can be distinguished, see, e.g., [1], [2]. Many TSOs correct infeasible outcomes of an unconstrained forward market via congestion redispatch, that is, by requesting counter transactions after gate closure. As an alternative, some operators employ locational marginal pricing (LMP) such as nodal or zonal pricing to directly influence the energy market during forward trade. Locational prices differ from the unconstrainedmarket price (determined by the lowest-cost producers) if congestion occurs, thus providing an incentive to schedule generation and load in a way that contributes to grid security.

In this paper, we compare the principles and effects of counter-trade and LMP-based congestion management on grid operation and market performance, particularly in terms of dispatch efficiency. Firstly, we indicate that only under particular conditions, both schemes can attain equal short-run social welfare. Secondly, we present a multiobjective modeling framework to decouple and analyze the effects of TSO and prosumer behavior on short-run network security and dispatch market efficiency. All results are illustrated using the widelyused IEEE 39-bus New-England benchmark network.

Notation: $\mathbb{R}$ and $\mathbb{R}^{n}, n=1,2, \ldots$, denote the field of real numbers and real-valued $n$-dimensional vectors, respectively. The operator $\operatorname{col}(\cdot)$ stacks its operands into a column vector; $\operatorname{diag}(\cdot)$ denotes a square matrix with its operands on the main diagonal and zeros elsewhere. Inequalities hold element-wise.

\section{Competitive Markets For Electrical EnERgy}

The main reason for deregulating the electricity market is to increase its efficiency through competition. This implies minimization of the long-run production costs, establishment of cost-reflective prices, while maximizing the sum of producer profit and consumer surplus (i.e., the social welfare), see, for instance, [3]. Yet, apart from energy supply/demand, few other aspects of the electrical power system lend themselves for competitive operation. Transmission is particularly unsuited for competition as a consequence of its natural monopoly character: duplicated lines are a waste of capital equipment and network expansion involves high investment costs. The monitoring, maintenance and construction of the European transmission system is therefore carried out by publiclyregulated TSOs.

To optimize system efficiency, markets for the supply and demand of electricity should be designed as competitive as possible. In competitive markets, producers adjust price and supply until the market reaches an equilibrium. Competitive market equilibriums can be short-run and long-run optimal, as defined below (see [3] for more details). 
Definition II.1 A short-run/dispatch-efficient market equilibrium is attained when marginal cost/benefit equals the market price and supply equals demand. This outcome optimizes social welfare given fixed productive resources. Under competition, the following conditions are necessary to guarantee that the market clears at the short-run optimal point: $(i)$ market liquidity is high, (ii) prosumers are price takers with strictly increasing marginal costs/strictly decreasing marginal benefit functions and (iii) prices are publicly available.

Definition II.2 A long-run/investment-efficient market equilibrium guarantees that the right (i.e., cost-minimizing) investments in production capacity have been made, and longrun social welfare has been maximized. Besides fulfilment of conditions $(i)-(i i i)$, this requires that (iv) there are no barriers for new competitors to enter and exit the market.

Note that in the above definitions, short-run/long-run refer to the completion of distinct market processes (that is, dispatch/investment planning, respectively) rather than to different time scales. Moreover, they relate to energy market efficiency in general rather than to the efficiency of transmission system operation and expansion. These aspects have significant influence on the fulfillment of conditions (i)-(iv) and thus on market performance, as is illustrated in Sect. III-IV.

\section{A. Ahead markets and uncertainty}

The operation of the electrical power grid, and thus the design of competitive markets for electricity, is complicated by significant physical restrictions. A lack of efficient storage mechanisms requires supply to meet fluctuations in demand all the time, whereas finite transmission-line capacities pose strict constraints on electrical power transmission.

Because of electricity's time-critical nature, real-time trading of energy (that is, trade for instantaneous delivery) is difficult. Instead, the bulk of electrical energy [MWh] is traded on forward markets such as the day-ahead market, i.e., the Power Exchange $(P X)$, where suppliers aim to maximize their profit while taking care of their expected internal energy balance (supply + demand + exchange $=0$ ). Together with long-term contracts and bilateral transactions, the PX outcome shapes the energy exchange schedules for the next operational day. Accordingly, all ahead-established energy transactions exist on paper only, as they define contracts to buy specific quantities of energy at a specified price with the supply set at a specified period in the future. There is no direct relation with the actual, TSO-monitored state of the electricity grid.

During real-time operation, grid users are unavoidably confronted with the physical limitations of electrical power and energy. As a result of imperfect predictions, the aheadestablished transactions will deviate from the actual supply and demand of energy, and unscheduled or infeasible power flows need to be counteracted immediately, to prevent network overloading. It is thus crucial for TSOs to design ahead-market schemes that are both as accurate and robust as possible, to minimize the need for real-time (fast and expensive) control

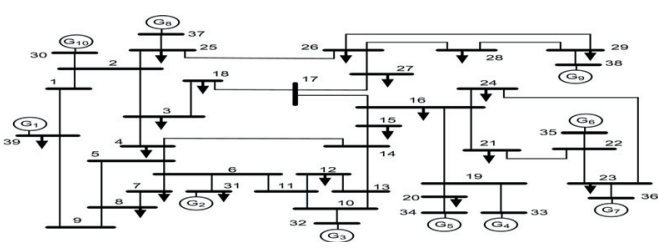

Fig. 1. The IEEE 39-bus New England test system.

effort, see, e.g., [4]. When market-based congestion management is the main focus, these methods should maximize social welfare while simultaneously taking limited network capacity and uncertainty of supply/demand into account. In the next section, we will discuss methods for a-priori congestion management in detail.

\section{Congestion Management}

We begin by introducing the power system modeling framework that is used throughout this paper. Let graph $G=(V, E, A)$ describe a transmission network, where $V=\left\{v_{1}, \ldots, v_{n}\right\}$ is a set of nodes/buses, $E \subseteq V \times V$ is a set of undirected edges/bus interconnections, and $A$ is a weighted adjacency matrix. The interconnection between bus $v_{i}$ and $v_{j}$ is denoted by $e_{i j}=\left(v_{i}, v_{j}\right)$. The adjacency matrix $A \in \mathbb{R}^{n \times n}$ satisfies $[A]_{i j}=-b_{i j} \neq 0 \Leftrightarrow e_{i j} \in E$ and $[A]_{i j}=0 \Leftrightarrow e_{i j} \notin E$, where $b_{i j}\left[\Omega^{-1}\right]$ is the susceptance of the line(s) associated with edge $e_{i j}$, see [1]. Self-connecting edges are not allowed (i.e., $e_{i i} \notin E$ ), such that $A$ has zeros on its main diagonal. The set of neighbors of a node $v_{i} \in V$ is denoted by $N_{i}:=\left\{v_{j} \in V \mid\left(v_{i}, v_{j}\right) \in E\right\}$; the corresponding indices are $I\left(N_{i}\right):=\left\{j \mid v_{j} \in N_{i}\right\}$. With each $e_{i j} \in E$, we associate a symmetric power-flow limit $\bar{p}_{i j}=\bar{p}_{j i}$.

The concepts discussed in this paper are illustrated using the widely-used IEEE 39-bus New England test system. Fig. 1 depicts the corresponding network topology, including priceelastic generators and price-inelastic loads. Line susceptance and load values for the network can be found in [5].

\section{A. The optimal power flow problem}

In conventional, regulated electrical power systems, the productive resources are owned by a small number of cooperating operators, such that power production can be scheduled in a centralized fashion, by solving an optimal power flow (OPF) problem. The OPF problem is instrumental to many marketbased congestion management schemes and is used to derive the LMP scheme later on.

To define the OPF problem, with each bus $v_{i} \in V$ we associate a singlet $\hat{p}_{i}[\mathrm{MW}]$ and a quadruplet $\left(p_{i}, \underline{p}_{i}, \bar{p}_{i}, J_{i}\right)$, where $p_{i}, \underline{p}_{i}, \bar{p}_{i}, \hat{p}_{i} \in \mathbb{R}, \underline{p}_{i}<\bar{p}_{i}$ and $J_{i}: \mathbb{R} \stackrel{\mathbb{R}}{\rightarrow}$ is a strictly convex, differentiable cost function. The values $p_{i}$ and $\hat{p}_{i}$ denote the reference values for power injections at each node into the network. Both $p_{i}$ and $\hat{p}_{i}$ can take positive as well as negative values, denoting production and consumption, respectively; the only difference is that in contrast to $\hat{p}_{i}$, the value $p_{i}$ has an associated cost/benefit function $J_{i}[€]$ and an interconnector capacity constraint $\underline{p}_{i} \leq p_{i} \leq \bar{p}_{i}$. We will thus refer to $p_{i}$ as the power from a price-elastic prosumer, and to $\hat{p}_{i}$ as the power from a price-inelastic prosumer. In 
the case of a positive $p_{i}$, the function $J_{i}$ represents the variable costs of production, while for negative values of $p_{i}$, it denotes the negated benefit of a consumer. Marginal production costs/benefits are denoted by $\nabla J_{i}[€ / \mathrm{MW}]$.

A lossless "DC power flow" model is employed to describe the power flows in the network for given nodal power injections. Under certain reasonable assumptions, this model is proven to be a relatively accurate approximation of the more complex "AC power flow" model, see, for instance, [1]. In particular, convexity of the DC power flow constraints is a crucial property that is exploited. With $\delta_{i}$ [rad] denoting the voltage phase angle at node $v_{i}$, the power flow in line $e_{i j} \in E$ is given by $p_{i j}=b_{i j}\left(\delta_{i}-\delta_{j}\right)=-p_{j i}$. If $p_{i j}>0$, power in the line $e_{i j}$ flows from node $v_{i}$ to node $v_{j}$. The power balance in a node yields $p_{i}+\hat{p}_{i}=\sum_{j \in I\left(N_{i}\right)} p_{i j}$. With $p:=$ $\operatorname{col}\left(p_{1}, \ldots, p_{n}\right), \hat{p}:=\operatorname{col}\left(\hat{p}_{1}, \ldots, \hat{p}_{n}\right), \delta:=\operatorname{col}\left(\delta_{1}, \ldots, \delta_{n}\right)$ and $\mathbf{1}_{n}:=[1, \ldots, 1]^{\top} \in \mathbb{R}^{n}$, the overall network balance condition is $p+\hat{p}=B \delta$, where $B:=A-\operatorname{diag}\left(A \mathbf{1}_{n}\right)$. The OPF problem is defined as follows.

\section{Problem III.1 Optimal power flow problem}

For any constant value of $\hat{p} \in \mathbb{R}^{n}$,

$$
\begin{array}{ll}
\operatorname{minimize}_{p, \delta} & \sum_{i=1}^{n} J_{i}\left(p_{i}\right) \\
\text { subject to } & p-B \delta+\hat{p}=0, \\
& \underline{p} \leq p \leq \bar{p}, \\
& b_{i j}\left(\delta_{i}-\delta_{j}\right) \leq \bar{p}_{i j}, \forall\left(i, j \in I\left(N_{i}\right)\right),
\end{array}
$$

where $\underline{p}=\operatorname{col}\left(\underline{p}_{1}, \ldots, \underline{p}_{n}\right), \bar{p}=\operatorname{col}\left(\bar{p}_{1}, \ldots, \bar{p}_{n}\right)$.

We will refer to a vector $p^{*}$ that solves the OPF problem as a vector of optimal power injections.

\section{B. Locational pricing}

Solving the OPF problem is one of the major operational (short-run) goals in a regulated power system. For liberalized, market-based power systems, the OPF problem is important due to its relation to the optimal nodal price problem (and similar LMP schemes) that is defined next.

In a market-based power system, different units are owned by separate parties and each of them acts autonomously to maximize its profit given the time-varying price for electricity. In other words, when a price-elastic unit at node $i$ receives the price for a certain period in the future, i.e. $\lambda_{i}[€ / \mathrm{MW}]$, it adjusts its scheduled prosumption $p_{i}$ to

$$
\tilde{p}_{i}=\Upsilon_{i}\left(\lambda_{i}\right):=\arg \min _{p_{i} \in\left[\underline{p}_{i}, \bar{p}_{i}\right]} J_{i}\left(p_{i}\right)-\lambda_{i} p_{i},
$$

where $\lambda_{i} p_{i}-J_{i}\left(p_{i}\right)$ is the profit/surplus of this particular unit. Since $J_{i}$ is a strictly convex function, the relation $\Upsilon_{i}: \mathbb{R} \rightarrow$ $\left[p_{i}, \bar{p}_{i}\right]$ defines a unique mapping from $\lambda_{i}$ to $\tilde{p}_{i}$ for any $\lambda_{i} \in \mathbb{R}$. For convenience, let $\Upsilon(\lambda):=\operatorname{col}\left(\Upsilon_{1}\left(\lambda_{1}\right), \ldots, \Upsilon_{n}\left(\lambda_{n}\right)\right)$. Note that if the capacity constraint $p_{i} \in\left[\underline{p}_{i}, \bar{p}_{i}\right]$ is ignored, it holds that $\lambda_{i}=\nabla J_{i}\left(\tilde{p}_{i}\right)$; since prosumers are price-takers/consider the price as given, they adjust prosumption until the corresponding marginal cost $\nabla J_{i}\left(p_{i}\right)$ equals the nodal price $\lambda_{i}$.

The foregoing shows that in a deregulated power system, the TSO cannot directly adjust nodal power injections to achieve a certain objective, e.g., to prevent congestion. Instead, it should
TABLE I

\begin{tabular}{lllllll}
\multicolumn{8}{c}{ GENERATOR PARAMETERS } \\
\cline { 1 - 2 } \cline { 5 - 7 } Bus $i$ & $c_{i}$ & $b_{i}$ & & Bus $i$ & $c_{i}$ & $b_{i}$ \\
\hline 30 & 0.8 & 30.00 & & 35 & 0.8 & 34.80 \\
31 & 0.7 & 35.99 & & 36 & 1.0 & 34.40 \\
32 & 0.7 & 35.45 & & 37 & 0.8 & 35.68 \\
33 & 0.8 & 34.94 & & 38 & 0.8 & 33.36 \\
34 & 0.8 & 35.94 & & 39 & 0.6 & 34.00 \\
\hline
\end{tabular}

provide market participants with price-based incentives for supporting such a system-wide goal. The operational goal in a nodal-pricing based power system is to determine the nodal price $\lambda_{i}$ for each node $i$ in the network, in such a way that short-run social welfare is maximized, while fulfilling both balance and network constraints. This optimal nodal pricing $(O N P)$ problem is defined as follows.

\section{Problem III.2 Optimal nodal prices problem}

For any constant value of $\hat{p} \in \mathbb{R}^{n}$,

$$
\begin{array}{ll}
\operatorname{minimize}_{\lambda, \delta} & \sum_{i=1}^{n} J_{i}\left(\Upsilon_{i}\left(\lambda_{i}\right)\right) \\
\text { subject to } & \Upsilon(\lambda)-B \delta+\hat{p}=0 \\
& b_{i j}\left(\delta_{i}-\delta_{j}\right) \leq \bar{p}_{i j}, \forall\left(i, j \in I\left(N_{i}\right)\right) .
\end{array}
$$

where $\lambda=\operatorname{col}\left(\lambda_{1}, \ldots, \lambda_{n}\right)$ is a vector of nodal prices.

We will refer to a vector $\lambda^{*}$ that solves the ONP problem with the term vector of optimal nodal prices. The OPF and ONP problems are related through Lagrange duality (see, e.g., [6]). It thus holds that $\Upsilon\left(\lambda^{*}\right)=p^{*}$, i.e., both problems implicitly define the vector of optimal nodal power injections that maximizes short-run social welfare.

In ONP-operated networks, the nodal price of electricity at a given time instant and bus reflects the least expensive way to increase the power flow to that particular node from the on-line generators while respecting all network constraints and system limits. Consequently, prices are identical throughout the network only if the transmission system has infinite capacity, or if OPF outcome $p^{*}$ yields no congestion. In the latter case, the network constraints have no effect on the forward market.

In what follows, we illustrate the nodal pricing concept using the New England test network. For this, the cost functions associated with the price-elastic generators at buses $i=30, \ldots, 39$ are parameterized as $J_{i}\left(p_{i}\right)=\frac{1}{2} c_{i} p_{i}^{2}+b_{i} p_{i}$, with $c_{i}, b_{i} \in \mathbb{R}$, yielding affine marginal costs or bids $\nabla J_{i}\left(p_{i}\right)=c_{i} p_{i}+b_{i}$. The values for $c_{i}$ and $b_{i}$ are listed in Table I. All generator capacity limits are set to $\underline{p}_{i}=0, \bar{p}_{i}=10$ (per unit, base value $100 \mathrm{MW}$ ). For simplicity, $\bar{p}_{i j}$ was set to infinite for all transmission lines, except for $e_{25,26}$. Fig. 2 shows the nodal prices for an uncongested (dashed line) and a congested (bars) network scenario, obtained by solving Prob. III.2 for $\bar{p}_{25,26}=\infty$ and $\bar{p}_{25,26}=1.5$, respectively. The corresponding optimal power injections are given in Table II. The unconstrained scenario yields $p_{25,26}=2.2326>1.5$. In the constrained scenario, only the power flow between node 25 to 26 is limited, yet trade is affected at all buses. This effect is typical for highly-interconnected meshed networks such as the 39-bus system. The constrained scenario leads to 


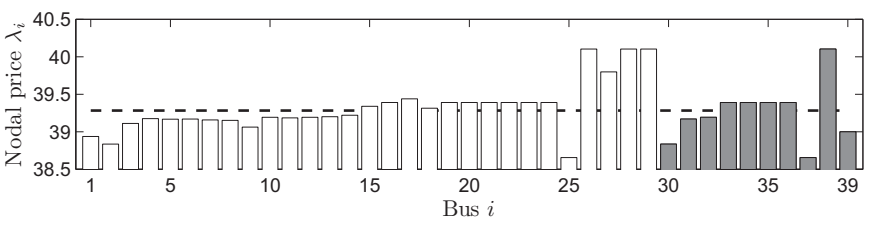

Fig. 2. Optimal nodal prices for an uncongested and a congested scenario.

TABLE II

OPTIMAL NODAL POWER INJECTIONS

\begin{tabular}{ll}
\hline $\bar{p}_{25,26}$ & $\left\{p_{30}^{*}, \ldots, p_{39}^{*}\right\}$ \\
\hline$\infty$ & $\{10.0,4.70,5.47,5.43,4.18,5.60,4.88,4.50,7.40,8.80\}$ \\
1.5 & $\{10.0,4.54,5.35,5.56,4.31,5.74,4.99,3.72,8.43,8.33\}$ \\
\hline
\end{tabular}

21 price areas, i.e., 21 clusters of buses with uniform nodal prices. This division of the network in price areas (or zones) is not static, but completely determined by the parameters of the ONP problem (such as the time-dependent bids $\nabla J_{i}$ ).

The 39-bus example shows that transmission-line restrictions may have varying effects on the nodal prices throughout the network. Congested lines do not support additional power flow, such that specific nodes (e.g., bus 38 in the simulation) can be cut off from the cheapest supplier in the network, which results in an increased nodal price (if the unconstrained market is taken as reference). Other prosumers (such as the ones at bus 25) can benefit from congestion, as the least expensive supply is distributed among a smaller number of accessible consumers. Still, since transmission-line restrictions narrow the domain over which the market is optimized, congestion always increases short-run cost while decreasing welfare. In the above simulation, for instance, if $\bar{p}_{i, j}=\infty$ for all lines, the optimized cost $J\left(p^{*}\right)$ and social welfare $\sum_{i=1}^{n} \lambda_{i}^{*} p_{i}^{*}-J_{i}\left(p_{i}^{*}\right)$ amount to $€ 2227.50$ and $€ 167.5423$, respectively, whereas the constrained scenario yields an optimal cost and welfare of $€ 2228.28$ and $€ 165.7812$, respectively.

As indicated by the example, transmission constraints can provide prosumers at badly accessible network locations with market power. If exercised, this power can result in nonoptimal local prices and market inefficiency. Adequate regulation of the TSO is therefore crucial to ensure that the operation and expansion of the transmission network contribute to welfare maximization, see Sect. IV.

\section{Curative congestion management}

LMP methods such as ONP explicitly confront market participants with network limitations, that is, with constraint (3c), during the ahead-trading stage. Consequently, when supply and demand bids $\nabla J_{i}\left(p_{i}\right)$ have been exchanged with the market, the optimal nodal prices for the next operational day can be computed in a single optimization run.

However, in practice, such preventive congestion management methods, i.e., methods that are employed before gate closure, may not be sufficient to guarantee satisfaction of security criteria during the operational day, see, e.g., [7]. Due to unexpected fluctuations in generation and load, or due to contingencies such as transmission-line faults, security criteria can be violated even if the market takes reasonable safety margins into account. Moreover, to limit the number of price areas in Europe, preventive methods currently only consider a subset of transmission restrictions. In contrast to ONP, where the clustering of buses with uniform prices may vary over time and is determined by the congested lines, the European energy market relies on a-priori fixed price areas (usually defined by political borders), within which Prob. III.2 is solved while ignoring internal transmission constraints.

TSOs can reduce the inter-area congestion risk by adjusting the forward-market's Available Transfer Capacity (ATC), which is allocated to market participants on the basis of periodically held auctions, see [8]. ATC values indicate the maximum amount of power that can be exchanged by adjacent price areas while ensuring system security. This is a considerable simplification of (3c), and it thus follows that ATC values are conditional upon the actual network-wide distribution of power injections. Since the exact nodal power injections are not known in advance, ATC values have to be computed based on expected dispatch scenarios. Moreover, due to the complexity of the European power system and the uncontrollable nature of electrical power flow, ATC values are so strongly interdependent that it is only possible to approximate them in a decentralized fashion.

ATC inaccuracy and intra-area transmission restrictions render curative congestion management, that is, congestion redispatch or counter trade after gate closure, indispensable for safe operation of the European transmission network. Normally, the TSO is the only buyer of curative counter transactions (i.e., supplementary transactions to recover secure network conditions), and their selection is usually based on merit-order criteria or long-term contracts, see [2]. Although there are many options for redispatch, here we only describe cost-based counter trade.

Let $\Delta p_{i} \in \mathbb{R}$ and $\Delta \delta_{i} \in \mathbb{R}$ be a power-injection and voltage-angle adjustment at bus $i$, measured with respect to the unconstrained-market outcome $p_{i}^{\mathrm{PX}}, \delta_{i}^{\mathrm{PX}} \in \mathbb{R}$. Suppose that elastic prosumers provide the TSO with knowledge of their adjustment cost/benefit, in the form of strictly convex functions $J_{i}^{\mathrm{CT}}: \mathbb{R} \rightarrow \mathbb{R}$. Then, the cost-based intra-area congestionredispatch procedure is formally defined as follows.

\section{Problem III.3 Curative congestion management}

1. The market solves Prob. III.2 while ignoring (3c) to find the uniform price vector $\bar{\lambda}^{\mathrm{PX}}=\operatorname{col}\left(\lambda^{\mathrm{PX}}, \ldots, \lambda^{\mathrm{PX}}\right) \in \mathbb{R}^{n}$, i.e., $\left(\bar{\lambda}^{\mathrm{PX}}, \delta^{\mathrm{PX}}\right):=\arg \min _{\{\lambda, \delta \text { s.t. }(3 \mathrm{~b})\}} \sum_{i=1}^{n} J_{i}\left(\Upsilon_{i}\left(\lambda_{i}\right)\right)$.

2. If $p^{\mathrm{PX}}:=\Upsilon\left(\bar{\lambda}^{\mathrm{PX}}\right)$ and $\delta^{\mathrm{PX}}$ violate (3c), the TSO employs congestion redispatch:

$$
\begin{array}{ll}
\operatorname{minimize}_{\Delta p, \Delta \delta} & \sum_{i=1}^{n} J_{i}^{\mathrm{CT}}\left(\Delta p_{i}\right) \\
\text { subject to } & \Delta p-B \Delta \delta=0 \\
& b_{i j}\left(\delta_{i}^{\mathrm{PX}}+\Delta \delta_{i}-\delta_{j}^{\mathrm{PX}}-\Delta \delta_{j}\right) \leq \bar{p}_{i j},
\end{array}
$$

for all $\left(i, j \in I\left(N_{i}\right)\right)$, where $\Delta p:=\operatorname{col}\left(\Delta p_{1}, \ldots, \Delta p_{n}\right)$ and $\Delta \delta:=\operatorname{col}\left(\Delta \delta_{1}, \ldots, \Delta \delta_{n}\right)$.

3. Given optimal redispatch vector $\Delta p^{*}$, the TSO pays $J_{i}^{\mathrm{CT}}\left(\Delta p_{i}^{*}\right)$ to prosumer $i$ as an incentive for adjusting its power injection to $p_{i}^{\mathrm{PX}}+\Delta p_{i}^{*}$. 
Next, suppose that the cost functions $J_{i}$ and capacities $\underline{p}_{i}, \bar{p}_{i}$ are time invariant ${ }^{1}$. Then, it holds that

$$
J_{i}^{\mathrm{CT}}\left(\Delta p_{i}\right)= \begin{cases}J_{i}\left(p_{i}^{\mathrm{PX}}+\Delta p_{i}\right)-J_{i}\left(p_{i}^{\mathrm{PX}}\right), & \Delta p_{i} \in\left[\underline{\Delta p}_{i}, \overline{\Delta p}_{i}\right] \\ \infty, & \Delta p_{i} \notin\left[\underline{\underline{\Delta p}}_{i}, \overline{\Delta p}_{i}\right]\end{cases}
$$

for all $i$, where $\underline{\Delta p} \underline{p}_{i}:=\underline{p}_{i}-p_{i}^{\mathrm{PX}}$ and $\overline{\Delta p}_{i}:=\bar{p}_{i}-p_{i}^{\mathrm{PX}}$. In this case, prosumers that are constrained on, i.e., increase production/decrease consumption, are exactly compensated for their increased variable cost/decreased variable benefit, whereas prosumers that are constrained off are financially indifferent between producing $p_{i}^{\mathrm{PX}}$ and participating in redispatch by reducing production with $\Delta p_{i}^{* 2}$.

Now consider the following proposition.

Proposition III.4 Let (5) hold. Then, Prob. III.2 and Prob. III.3 (i) define identical nodal power injections and prosumer costs, but (ii) differ in terms of short-run social welfare.

Proof. (i) From (5) and the construction of $p_{i}^{\mathrm{PX}}, \delta_{i}^{\mathrm{PX}}, \Delta p_{i}^{*}$ and $\Delta \delta_{i}^{*}$, it straightforwardly follows that $p^{\mathrm{PX}}+\Delta p^{*}$ and $\delta^{\mathrm{PX}}+\Delta \delta^{*}$ solve the OPF problem. Thus, the power injections and angles defined by Prob. III.3 satisfy $p_{i}^{\mathrm{PX}}+\Delta p_{i}^{*}=p_{i}^{*}$ and $J_{i}\left(p_{i}^{\mathrm{PX}}\right)+J_{i}^{\mathrm{CT}}\left(\Delta p_{i}^{*}\right)=J_{i}\left(p_{i}^{\mathrm{PX}}+\Delta p_{i}^{*}\right)=J_{i}\left(p_{i}^{*}\right)$ for all $i$. (ii) In case of ONP/Prob. III.2, the income and cost of the producers (or, equivalently, the cost and benefit of the consumers) at node $i$ equal $\lambda_{i}^{*} p_{i}^{*}$ and $J_{i}\left(p_{i}^{*}\right)$, respectively, whereas in case of Prob. III.3, the producer income and cost amount to $\lambda^{\mathrm{PX}} p_{i}^{\mathrm{PX}}+J_{i}^{\mathrm{CT}}\left(\Delta p_{i}^{*}\right)$ and $J_{i}\left(p_{i}^{*}\right)$, respectively. Thus, the social welfare is $\sum_{i=1}^{n}\left\{\lambda_{i}^{*} p_{i}^{*}-J_{i}\left(p_{i}^{*}\right)\right\}$ for ONP and $\sum_{i=1}^{n}\left\{\lambda^{\mathrm{PX}} p_{i}^{\mathrm{PX}}-J_{i}\left(p_{i}^{\mathrm{PX}}\right)\right\}$ for cost-based redispatch. These values are not identical, except for $p^{\mathrm{PX}}=p^{*}$.

Next, we describe a simple curative counter transaction for the 39-bus test network to illustrate the above result and its consequences for the TSO. Recall from Sect. III-B that the optimal vector of nodal power injections for the unconstrained market (corresponding to $\lambda^{\mathrm{PX}}=39.2817$, see Table II) violates the transmission-line constraints. The TSO can ask market participants what compensation they are willing to accept to adjust their prosumption in such a way that the power balance is maintained and the flow $p_{25,26}$ is lowered to $\bar{p}_{25,26}=1.5$. These conditions are met, e.g., if the generators at buses 37 and 38 decrease and increase their power injections with 0.7326 , respectively. Since the generator at node 37 reduces its production with respect to $p_{37}^{\mathrm{PX}}$, it pays $J_{37}\left(p_{37}^{\mathrm{PX}}\right)-J_{37}\left(p_{37}^{\mathrm{PX}}-0.7326\right)=€ 41.6527$ to the TSO, whereas the TSO pays $J_{38}\left(p_{38}^{\mathrm{PX}}+0.7326\right)-J_{38}\left(p_{38}^{\mathrm{PX}}\right)=€ 42.5723$ for increasing the generation at bus 38 . Thus, the cost associated with this pair of bilateral transactions is $€ 0.9195$.

Under assumption (5), it can be shown that there is no other feasible pair of bilateral transactions that yields lower

\footnotetext{
${ }^{1}$ This assumption is nontrivial: depending on the generators and the time frame involved, cost functions and capacities will generally change over time. Consider for instance start/stop times that are irrelevant during forward trade, but that prevent redispatch of the corresponding sources after gate closure.

${ }^{2}$ Note that a small $\varepsilon[€]$ term may be added to $J_{i}^{\mathrm{CT}}\left(\Delta p_{i}\right)$ to provide prosumers with a strictly positive incentive for redispatch.
}

redispatch costs than the one described above. However, if the selection of counter transactions is not limited to a subset of the generators, the overall prosumption costs can be lowered to those of the OPF solution (without affecting short-run social welfare, which is $€ 167.5423$ regardless of the redispatch), yielding minimum costs for the TSO, i.e., $€ 0.7758$. Still, by construction, a constrained market (e.g., Prob. III.2) cannot outperform the corresponding unconstrained market (e.g., Prob. III.2 without constraints (3c)), such that the TSO generally suffers losses from counter trade. In case of efficient behavior, the TSO can usually socialize and recover these congestion redispatch costs via a system-wide transmission tariff. Note that this is in contrast to ONP-based networks, where the forward market price may be non-uniform and explicitly confronts prosumers, without intervention by the TSO, with both energy and location-specific transmission costs.

\section{MARKET EFFICIENCY AND NETWORK SECURITY}

Since Prob. III.2 implicitly defines the vector of power injections that maximizes short-term social welfare, one might conclude that ONP, and, at best, also OPF-based redispatch, lead to a dispatch efficient market equilibrium. However, since the vector of optimal power injections $p^{*}$ is a function of network parameters $B$ and $\bar{p}_{i j}$, it is hard to draw firm conclusions on dispatch efficiency without analyzing how these network parameters are obtained. $B, \bar{p}_{i j}$ and ATC values are stochastic variables, and only the TSO has sufficient information to predict their value in a reliable fashion ${ }^{3}$. Since the TSO has a natural monopoly on transmission, regulation is required to avoid possible abuse of his powerful position in the market. The monitoring of line-susceptance prediction quality is straightforward, as real-time measurements allow for a-posteriori comparison with the TSO's expectations. This is not the case for transfer-capacity (or ATC) profiles, which consist of both estimated thermal transmission-line limits and safety margins that are selected at the TSO's discretion.

The above described non-transparency provides network operators with the possibility to exploit flow-capacity estimation in their own benefit, see, e.g., [9]. Naturally, TSOs tend to minimize the chance of network overloading by choosing security margins as large as possible, whereas prosumers demand maximum transmission capacity, i.e., minimum margins, to optimize social welfare. In what follows, we provide a way to model this trade-off between conflicting short-run security and market efficiency objectives as a multiobjective optimization problem, see for instance [10].

Let $f_{i j}[\mathrm{MW}]$ and $\Delta f_{i j}[\mathrm{MW}]$ be the thermal limit (determined by external factors such as weather conditions) and the security margin (set by the TSO) of transmission line $e_{i j}$, respectively, such that $\bar{p}_{i j}:=f_{i j}-\Delta f_{i j}$. Note that $0 \leq f_{i j}$ and $0 \leq \Delta f_{i j} \leq f_{i j}$. Next, let $f:=\operatorname{col}\left(\left\{f_{i j} \mid e_{i j} \in E\right\}\right)$ and $\Delta f:=\operatorname{col}\left(\left\{\Delta f_{i j} \mid e_{i j} \in E\right\}\right)$, and consider an objective function $S(\Delta f)[€]$ that represents the TSO's financial risk of

\footnotetext{
${ }^{3}$ Note that also in the long-run, the TSO is the only market actor able to adjust $B$ and $\bar{p}_{i j}$ by investing in the transmission infrastructure.
} 
congestion, overloading and line outages/damage associated with a particular set of safety margins $\Delta f$. For simplicity, assume that $S(\Delta f)$ is decreasing in all $\Delta f_{i j}$, and that $S(\Delta f) \rightarrow 0$ if $\Delta f_{i j} \rightarrow f_{i j}$ for all $\left(i, j \in I\left(N_{i}\right)\right)$. Moreover, let $J(p):=\sum_{i=1}^{n} J_{i}\left(p_{i}\right)[€]$ map the vector of nodal power injections $p \in \mathbb{R}^{n}$ to the corresponding total prosumption cost/benefit. Now consider the following problem.

Problem IV.1 Power system security/efficiency trade-off For any constant value of $\hat{p}$ and $f$,

$$
\min _{\Delta f, \lambda, \delta}[S(\Delta f) \quad J(\Upsilon(\lambda))]^{\top}
$$

subject to $0 \leq \Delta f \leq f$, (3b)-(3c), where $\bar{p}_{i j}:=f_{i j}-\Delta f_{i j}$.

Multiobjective optimization problems such as the one above have an infinite number of solutions, as there are infinite ways to trade off two or more conflicting goals. A feasible point $\left(\Delta f^{*}, \lambda^{*}, \delta^{*}\right)$ lies within the solution space of Prob. IV.1 if and only if it is Pareto optimal, i.e., if all points corresponding to lower security costs $S$ (or lower prosumption cost $J$ ) yield larger $J$ (or $S$ ). One way to solve Prob. IV.1 is to construct a single composite objective function $C(\Delta f, \lambda):=r S(\Delta f)+$ $J(\Upsilon(\lambda))$, with positive scalar weight $r \in \mathbb{R}$, and solve

$$
\min _{\Delta f, \lambda, \delta} C(\Delta f, \lambda)
$$

subject to $0 \leq \Delta f \leq f$, (3b) and (3c). Each outcome of the composite optimization problem corresponds to a particular trade-off that is characterized by $r$. Thus, the full set of solutions (i.e., the trade-off surface/Pareto frontier) is found by evaluating (7) for all $r \in[0, \infty)$.

In what follows, we illustrate the above concept with the 39-bus network. For simplicity, let $\bar{p}_{i j}$ be infinite for all lines except $e_{25,26}$, and let $S(\Delta f):=\left(\Delta f_{25,26}-f_{25,26}\right)^{2}$, where $f_{25,26}=1.5$. Fig. 3 shows the resulting Pareto frontier in the $J(\cdot), S(\cdot)$ plane. Clearly, minimal network costs $S(\Delta f)$ are attained for $r \rightarrow \infty$. This ratio corresponds to a security/efficiency trade-off that is completely in favor of the TSO (i.e., line $e_{25,26}$ is not loaded at all). Social welfare, on the other hand, is maximized for $r=0$, in which case $\Delta f_{25,26}=0$ and transmission risks are high.

Fig. 3 shows that $r$ can have significant effects on market and system performance. The regulator can choose any positive value for $r$ that is in accordance with (inter)national legislation, to trade off security against market efficiency in a way that best fits his priorities. As an example of a point of operation that the regulator can pursue, we mention the egalitarian solution $P$, see [10]. A power system is operated in an egalitarian fashion if the regulator appreciates system safety and market performance to an equal extent, yielding an outcome of Prob. IV.1 that satisfies $\frac{\mathrm{d}}{\mathrm{d} r} S\left(\Delta f^{*}(r)\right)=$ $-\frac{\mathrm{d}}{\mathrm{d} r} J\left(p^{*}(r)\right)$. As shown in Fig. 3, the egalitarian solution of Prob. IV.1 is the point $P=(S(\cdot), J(\cdot))$ where the tangent to the Pareto frontier has a slope $\frac{\mathrm{d} S}{\mathrm{~d} J}$ of -1 .

The above modeling framework provides a transparent way to decouple and analyze the effects of TSO behavior, prosumer bids and network regulation on short-run market efficiency,

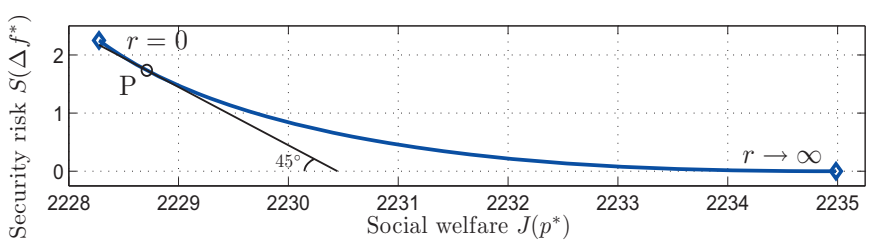

Fig. 3. Security/efficiency Pareto front for the 39-bus example network.

independent of the applied congestion management scheme. Moreover, it explicitly associates network reliability (measured in terms of line margins $\Delta f$ ) with the expected costs for the network operator. Note that even though it is difficult to predict network security costs a priori, there are many observation-based methods for approximating them empirically, see, e.g., [11] and the references therein.

\section{Conclusions}

In this paper, the differences between locational pricing and cost-based congestion redispatch were analyzed, followed by an assessment of their effects on grid operation. It was shown that although optimal nodal pricing and cost-based congestion redispatch yield identical power injections, they are not equivalent in terms of social welfare. Moreover, a multiobjective modeling framework was presented to decouple and analyze the effects of TSO/regulator and prosumer behavior on shortrun power system security and market efficiency.

\section{ACKNOWLEDGEMENTS}

This research is part of the EOS-Regelduurzaam and E-Price projects that are funded by SenterNovem/Agentschap NL and the European Community Framework Programme 7, respectively.

\section{REFERENCES}

[1] R. D. Christie, B. F. Wollenberg, and I. Wangensteen, "Transmission management in the deregulated environment," Proceedings of the IEEE, vol. 88, no. 2, pp. 170-195, 2000.

[2] E. Bompard, P. Correia, G. Gross, and M. Amelin, "A comparative analysis of congestion management schemes under a unified framework," IEEE Power Engineering Review, vol. 22, no. 11, pp. 59-60, Nov. 2002.

[3] S. Stoft, Power System Economics: Designing Markets for Electricity. Kluwer Academic Publishers, 2002.

[4] P. P. J. van den Bosch, A. Jokić, J. Frunt, W. L. Kling, F. Nobel, P. Boonekamp, W. de Boer, R. M. Hermans, and A. Virag, "Pricebased control of ancillary services for power balancing," European Transactions on Electrical Power, 2010, in press.

[5] M. A. Pai, Power System Stability by Lyapunov's Method. Amsterdam: North-Holland Publishing Company, 1981.

[6] A. Jokić, "Price-based optimal control of electrical power systems," Ph.D. dissertation, Eindhoven University of Technology, Eindhoven, The Netherlands, 2007.

[7] "An evaluation of preventive countertrade as a means to guarantee firm transmission capacity," European Transmission System Operators (ETSO), Tech. Rep., April 2005.

[8] "Net transfer capacities and available transfer capacities in the internal market of electricity in Europe," European Transmission System Operators (ETSO), Tech. Rep., March 2000.

[9] J.-M. Glachant and V. Pignon, "Nordic congestion's arrangement as a model for Europe? Physical constraints vs. economic incentives," Utilities Policy, vol. 13, no. 2, pp. 153-162, 2005.

[10] Y. Sawaragi, H. Nakayama, and T. Tanino, Theory of Multiobjective Optimization, ser. Mathematics in Science and Engineering. Orlando, FL: Academic Press Inc., 1985, vol. 176.

[11] A. A. Chowdhury and D. O. Koval, "Quantitative transmission-systemreliability assessment," IEEE Transactions on Industry Applications, vol. 46, no. 1, pp. 304-312, Jan.-Feb. 2010. 